\title{
Comparison of the Effects of a Brand-name Drug and Its Generic Drug on the Quality of Life of Alzheimer's Disease Patients
}

\author{
Mikio Sakakibara ${ }^{1,2,3}$, Mitsuhiko Kido ${ }^{1,2,4}$, Jun Kuribayashi ${ }^{2,4}$, Hiroshi Okada ${ }^{1,2}$, Ataru Igarashi ${ }^{5}$, Hiroyuki Kamei ${ }^{3}$, \\ Toshitaka Nabeshima ${ }^{1,4}$ \\ ${ }^{1}$ NPO Japanese Drug Organization of Appropriate Use and Research, ${ }^{2}$ Sugi Pharmacy Co., Ltd., ${ }^{3}$ Pharmaceutical Hospital Laboratory, \\ Faculty of Pharmacy, Meijo University, ${ }^{4}$ Department of Regional Pharmaceutical Care and Sciences, Faculty of Pharmacy, Meijo University, \\ Nagoya, ${ }^{5}$ Department of Drug Policy and Management, Graduate School of Pharmaceutical Sciences, The University of Tokyo, Tokyo, \\ Japan
}

\begin{abstract}
Objective: The pharmacological effects of generic (GE) donepezil are the same as Aricept, its brand-name counterpart. However, little is known as to whether these two drugs provide the same quality of life (QOL). The study subjects were patients with Alzheimer's disease who were taking donepezil hydrochloride tablets, and were selected by visiting either the local pharmacies or the patients' homes. We chose the brand-name drug Aricept and its GE form donepezil to investigate, from a long-term caregiver's perspective, the influence of both drugs on the patients' QOL.

Methods: An EuroQol-5 Dimension (EQ-5D) was used to assess the QOL of patients with Alzheimer's disease, before and after various Aricept and/or donepezil regimens. Patients were divided into four groups: first time users of Aricept ( $n=43$ ), first time users of GE donepezil ( $n=45)$, users refilling previous prescriptions of Aricept ( $n=51$ ), and users switching from Aricept to GE donepezil $(n=51)$.

Results: The average change in the $E Q-5 D$ utility indices rose significantly in the patients starting a new regimen of Aricept and its GE drug. The patients continuing an existing regimen of Aricept showed no significant differences, even after Aricept was switched to a GE drug.

Conclusion: The QOL of patients starting a new regimen of Aricept and its GE drug improved. The QOL was maintained upon switching to the GE drug form.
\end{abstract}

KEY WORDS: Alzheimer disease; Donepezil hydrochloride; Generic drugs; Quality of life; EuroQol-5 Dimension.

\section{INTRODUCTION}

Now that Japan has become an ultra-aging society, there is a growing need to provide care to patients in their homes. Pharmacists working at community pharmacies support this home-based medical care. Elderly patients are generally treated with drugs, both at home and in nursing care facilities. However, we are seeing an increase in medical treatment risks associated with drug therapies that are unique to elderly patients, such as a decline in swallowing function and difficulty managing and administrating mul-

\footnotetext{
Received: January 21, 2015 / Revised: February 23, 2015

Accepted: February 24, 2015

Address for correspondence: Mikio Sakakibara, BS

NPO Japanese Drug Organization of Appropriate Use and Research, Headquarters, Department of Regional Pharmaceutical Care and

Sciences, Faculty of Pharmacy, Meijo University, 3-1509, Omoteyama,

Tenpaku-ku, Nagoya, Aichi Prefecture 468-0073, Japan

Tel: +81-52-834-5963, Fax: +81-52-834-5963

E-mail: sakakibara606@gmail.com
}

tiple prescription drugs. There is a need for more extensive measures to cope with these risks.

The morbidity rate of dementia among the elderly Japanese aged over 65 years is estimated to be $15 \%$, and the number of individuals suffering from dementia is estimated to be approximately 4.62 million. ${ }^{1)}$ Patients in Japan with mild cognitive impairment (MCI) see a decline in their cognitive function; however, this does not notably hamper their daily living. The morbidity rate of $\mathrm{MCI}$ is estimated to be $13 \%$, and the estimated number of patients as of 2012 was 4.00 million. ${ }^{1)}$ To continue to adopt measures to cope with dementia, we need to understand dementia accurately and improve care and medical treatments. At present, the goal is to allow elderly people including dementia patients to lead high-quality lives. In response to these challenges, the Japanese Ministry of Health, Labour and Welfare created a Five-Year Plan for Promotion of Measures Against Dementia, called the Orange Plan.

(a) This is an Open-Access article distributed under the terms of the Creative Commons Attribution Non-Commercial License (http://creativecommons.org/licenses/by-nc/4.0) which permits unrestricted non-commercial use, distribution, and reproduction in any medium, provided the original work is properly cited 
Programs were launched in 2013 financial year. It is important to create a setup in each region to implement strategies to enable (1) early and appropriate diagnosis and responses, and (2) provisions of support to the patients and their families based on accurate knowledge and understanding of dementia. ${ }^{2)}$

Community pharmacists promote the use of generic (GE) drugs to reduce medical spending, to ensure the proper use of GE drugs, and to maintain the quality of life (QOL) of patients and their families. In promoting the use of GE drugs, it is also imperative that the drugs be selected through appropriate and evidence-based evaluations.

We conducted an investigation, targeting Alzheimer's disease patients who were given donepezil hydrochloride tablets, either by visiting community pharmacies or at home, to study how the use of two drugs - a brand-name drug (Aricept, Eisai Co., Tokyo, Japan) and its GE drug (donepezil)-affected the patients' QOL. The investigation was conducted from the caregivers' perspectives. We also investigated whether the QOL of the group who took Aricept continuously was maintained, even if Aricept was switched to the GE drug. Naglie et al. ${ }^{3)}$ used three methods to evaluate the QOL of Alzheimer's disease patients, namely, the Health Utilities Index Mark III, Quality of Well-Being, and EuroQol-5 Dimension (EQ-5D), and compared their findings. The authors reported that evaluations by EQ-5D were the most reliable, valid, and feasible. Kohno et $a l^{4)}$ have reported that because the number of elderly dementia patients will continue to increase in Japan, the importance of using patient QOL as an indicator would rise; obtaining guidelines for care, based on the evaluation of EQ-5D whose reliability and validity were confirmed by research, carried great significance.
Using the EQ-5D assessment, we investigated the QOL of Alzheimer's disease patients who took the brand-name drug (Aricept) or its GE drug (donepezil). QOL was assessed initially (T1) and again 4 months later (T2) in patients: starting a new regimen of Aricept, starting a new regimen of the GE drug, continuing to take Aricept, or switching to the GE drug from Aricept. Patients who started a new regimen of Aricept or its GE drug showed an average increase in QOL 4 months after the initial screening. Patients who continued a regimen of Aricept and those who switched to the GE drug did not show significant difference in QOL.

\section{METHODS}

Investigations were carried out at sixteen pharmacies from January to December 2013. From the patients involved, we obtained written consent describing their intention to participate in the investigation. We divided 190 patients (73 males and 117 females, aged 68-98 years) who were taking donepezil hydrochloride tablets (including orally disintegrating tablets) into the following four groups: The NA group (patients starting a new regimen of Aricept $\mathrm{T}, 43$ patients); the NG group (patients starting a new regimen of Aricept's GE drug, 45 patients); the AA group (patients continuing a regimen of Aricept: 51 patients); the AG group (patients continuing a regimen of Aricept, but switched to its GE drug, 51 patients) (Fig. 1).

The influence of Aricept and its GE drug on QOL was analyzed using the utility index obtained from the Japanese-language edition of EQ-5D. We asked the same caregivers how they evaluated the QOL of each patient on the start date (primary investigation) and four months later (secondary investigation) (Fig. 1). The Japanese-language

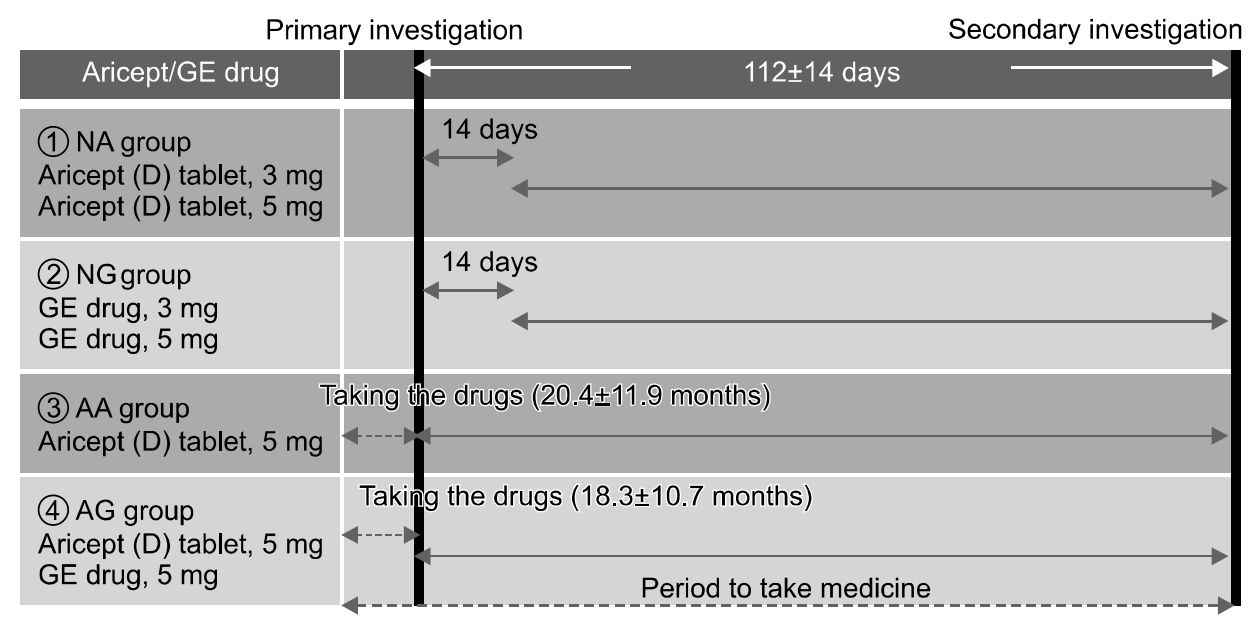

Fig. 1. New-ACT (NA) group: patients who started a new regimen of Aricept (43 patients). New-GE (NG) group: patients who started a new regimen of Aricept's GE drug (45 patients).

ACT-ACT (AA) group: patients who continued an existing regimen of Aricept (51 patients).

ACT-GE (AG) group: patients who continued an existing regimen of Aricept, but switched to the GE drug (51 patients). 


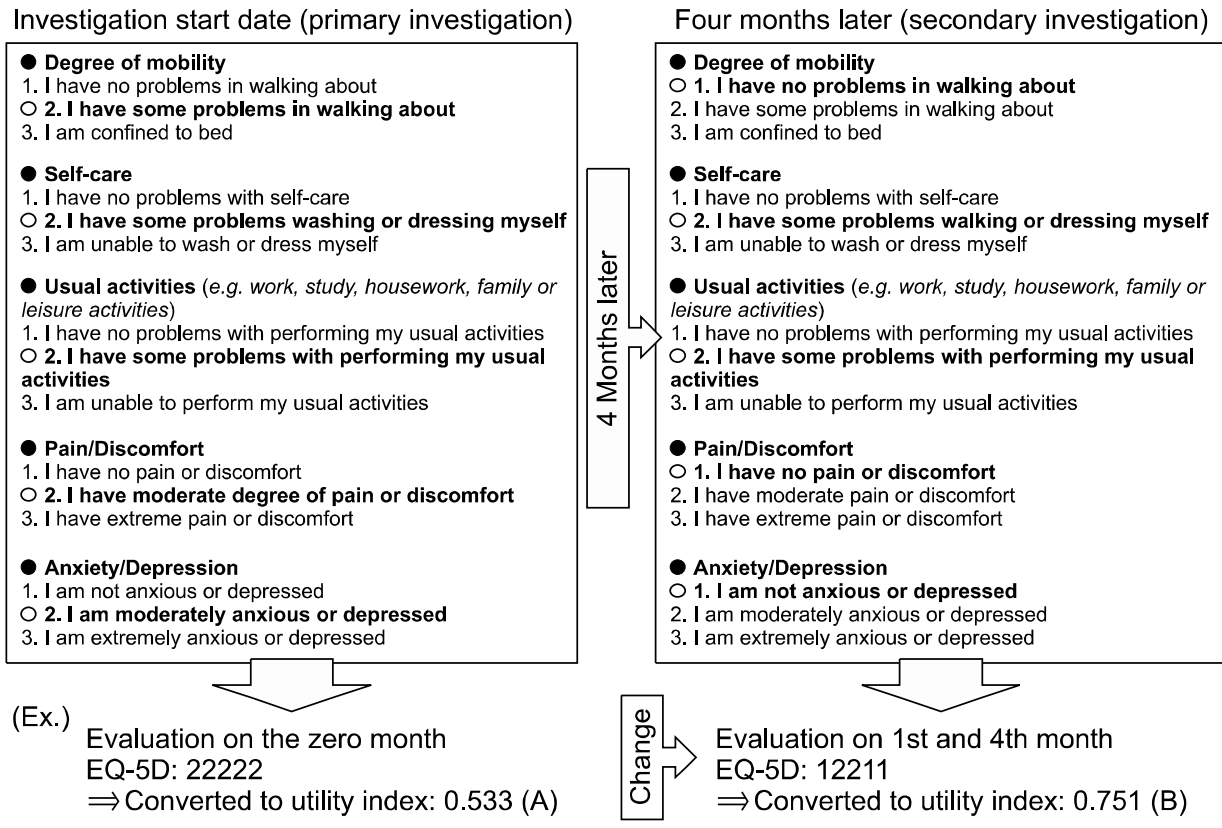

Amount of change $(Q O L$ improvement $)=$ Rise in utility index $(B)-(A)=0.751-0.533=0.218$
Fig. 2. Questions posed in EuroQol-5 Dimension (EQ-5D) and amount of change. QoL, quality of life. edition of EQ-5D divides a person's state of health into five dimensions (mobility, self-care, usual activities, pain/discomfort, and anxiety/depression) and evaluates each on a 3-point scale (no problems, some problems, and extreme problems). The answers obtained from caregivers are converted into a single yardstick - a utility index - using the Japanese-language edition of the utility conversion table (Fig. 2). According to the utility index, "best imaginable state of health" is defined as 1 and death is defined as 0 . Using this index, evaluations can be made in cases of relative performance differences according to sector and where comprehensive assessment of therapeutic effects is problematic, such as "although discomfort and anxiety disappeared, the degree of mobility worsened". 5)

This study was designed as prospective observational study. In this study, due to ethical considerations, the subjects were not allocated to various groups in advance. They were divided into four groups and compared at the end of the investigational period. In all four groups, the subjects continued to receive their usual treatments and dosing guidance. In eleven studies, the difference in the EQ-5D utility index showing clinical differences was reported to be 0.074 (range, 0.011-0.140). ${ }^{6}$ Therefore, we determined that significant differences were seen if the difference in utility indices exceeded \pm 0.1 . We have received an approval to use the EQ-5D.

For statistical analysis, we used the Wilcoxon signed-ranks test to identify any significant differences in the mean EQ-5D utility indices among the four groups and the Mann-Whitney rank-sum test to analyze for significant differences in the average amount of change in the EQ-5D utility indices in the four groups.

In accordance with the ethics guidelines of epidemiological studies, consent was obtained orally from patients (or family members/other caregivers in cases where expression of intent was difficult for patients themselves) regarding their participation in the research and was recorded in writing. The Ethics Committee of NPO Japanese Drug Organization of Appropriate Use and Research approved the research protocol (2013\#1). All data analyses were conducted after names were made anonymous.

\section{RESULTS}

Table 1 shows the backgrounds of the patients in the four groups who took both Aricept and the GE drug, including their mean age, male-female ratio, frequency of dosing guidance, and the pre-investigation administration period. The mean age was 77.1 years in the NA group, 80.3 years in the NG group, 83.3 years in the AA group, and 80.4 years in the AG group. The male-female ratio (male : female) was $17: 26$ in the NA group, $19: 26$ in the NG group, $16: 35$ in the AA group, and $20: 31$ in the AG group. The frequency of dosing guidance was 5.02 in the NA group, 5.16 in the NG group, 4.63 in the AA group, and 4.75 in the AG group. No major differences were seen 
Table 1. Background of the patients in the 4 groups taking Aricept and its generic (GE) drug

\begin{tabular}{lcccc}
\hline \multirow{2}{*}{ Variable } & \multicolumn{4}{c}{ Group } \\
\cline { 2 - 5 } & NA & NG & AA & AG \\
\hline Participation, $\mathrm{n}$ & 68 & 69 & 65 & 67 \\
Dropout, $\mathrm{n}$ & 25 & 24 & 14 & 16 \\
Patient, $\mathrm{n}$ & 43 & 45 & 51 & 51 \\
Mean age, yr & 77.1 & 80.3 & 83.3 & 80.4 \\
Mean nursing care level* & 1.6 & 1.8 & 2.1 & 1.9 \\
Male : female, $\mathrm{n}(\%)$ & $17: 26$ & $19: 26$ & $16: 35$ & $20: 31$ \\
& $(40: 60)$ & $(42: 58)$ & $(31: 69)$ & $(39: 61)$ \\
Dosing guidance, $\mathrm{n}$ & 5.02 & 5.16 & 4.63 & 4.75 \\
Period of administration & 0 & 0 & 20.4 & 18.3 \\
prior to investigation (mo) & \multicolumn{5}{c}{} \\
\hline
\end{tabular}

NA, patients starting a new regimen of Aricept; NG, patients starting a new regimen of Aricept's generic drug; AA, patients continuing a regimen of Aricept; $A G$, patients continuing a regimen of Aricept, but switched to its GE drug.

*Between 1 and 5 based on assessment of care requirements.

in the male-female ratio and frequency of dosing guidance. The period of administration prior to the investigation of the two groups that continued to take Aricept was 20.4 months in the AA group and 18.3 months in the AG group, showing no significant differences. There was no difference in the effect of donepezil against QOL between men and women.

Table 2 shows the average EQ-5D utility indices assessed in the four groups. The utility indices of the NA and NG groups showed a significant difference $(p<0.01)$ between the values calculated during the primary and secondary investigations. However, the utility indices of the AA group and the AG group showed no significant differences between the evaluations made during the primary and secondary investigations. Fig. 3 shows the average change in the EQ-5D utility indices of the four groups. The average change was calculated by subtracting the evaluation during the secondary investigation from the evaluation during the primary investigation. The average change between the NA and NG groups was over 0.1 , but the average change between the AA and AG groups was less than 0.1 . There was a significant difference $(p<0.01)$ in the average change between the NA-AA groups and between the NG-AG groups. On the other hand, no significant differences were seen in the average change between the NA-NG groups and the AA-AG groups.

\section{DISCUSSION}

\section{Comparison of the Effects between the Brand Name and GE Drug}

The current investigation analyzed QOL, using EQ-5D,
Table 2. Utility index of EuroQol-5 Dimension of the patients in the 4 groups taking Aricept and its generic (GE) drug

\begin{tabular}{lcccc}
\hline \multirow{2}{*}{ Variable } & \multicolumn{4}{c}{ Group } \\
\cline { 2 - 5 } & NA & NG & AA & AG \\
\hline Patient, $n$ & 43 & 45 & 51 & 51 \\
Primary & 0.583 & 0.551 & 0.512 & 0.477 \\
& $(0.189)$ & $(0.207)$ & $(0.237)$ & $(0.243)$ \\
Secondary & 0.685 & 0.720 & 0.516 & 0.501 \\
& $(0.200)$ & $(0.224)$ & $(0.230)$ & $(0.244)$ \\
Average change & $0.102^{*}$ & $0.169^{*}$ & 0.004 & 0.024 \\
\hline
\end{tabular}

Values are presented as number only or mean (standard deviation).

NA, patients starting a new regimen of Aricept; NG, patients starting a new regimen of Aricept's generic drug; AA, patients continuing a regimen of Aricept; $A G$, patients continuing a regimen of Aricept, but switched to its GE drug. ${ }^{*} p<0.01$ vs. primary.

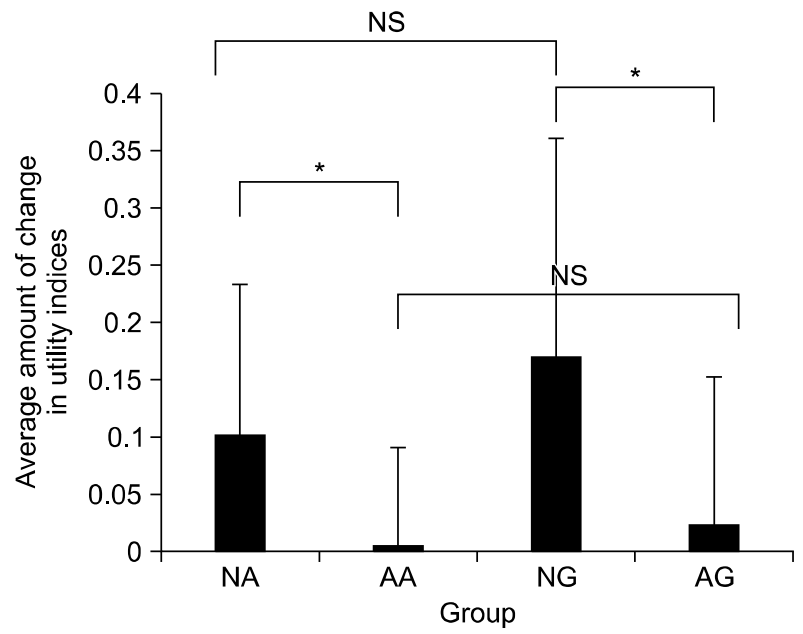

Fig. 3. Average change in EQ-5D's utility indices of the patients in the 4 groups taking Aricept and its generic drug (mean \pm standard deviation).

Average change=(evaluation during the secondary investigation) - (evaluation during the primary evaluation)

A significant difference $\left({ }^{*} p<0.01\right)$ was assessed between the NA and AA groups, and between the NG and $A G$ groups. No significant differences were assessed between the NA and NG groups, or between the $A A$ and $A G$ groups.

$N A$, patients starting a new regimen of Aricept $T$; NG, patients starting a new regimen of Aricept's generic drug; AA, patients continuing a regimen of Aricept; $A G$, patients continuing a regimen of Aricept, but switched to its GE drug; EQ-5D, EuroQol-5 Dimension.

in Alzheimer's disease patients who were taking brand name Aricept or the GE form, donepezil. The patients that began a new course of drug treatment experienced a similar increase in QOL after 4 months of treatment, regardless of which drug they used (NG, NA). Patients continuing to take Aricept (AA) or those that switched from Aricept to the GE form, donepezil (AG), experienced no significant change in QOL after four months of treatment. 
These results suggested that the GE drug has similar effects to the brand-name drug regardless of beginning, continuing, or switching drug treatments.

The results showed a statistically significant rise, exceeding 0.1 , in the utility index of EQ-5D for the groups that started a new drug regimen (the NA and NG groups). These findings suggested that a new regimen of donepezil hydrochloride could improve the QOL of Alzheimer's disease patients. Our results supported the previous report of Asada et al. ${ }^{7)}$ that showed a significant difference in clinical utility value, exceeding 0.1 , in the utility index of EQ-5D evaluated by caregivers. Although no significant rise was assessed in the groups that continued to take donepezil hydrochloride (AA and AG groups), the average change rose slightly in the AA group (0.004), and the AG group (0.024), which suggested that their QOL was being maintained by administration of donepezil hydrochloride.

Since a significant difference $(p<0.01)$ was assessed between the NA-AA groups and between the NG-AG groups, we concluded that starting a new regimen of donepezil hydrochloride (Aricept or its GE drug) leads to improved QOL in Alzheimer's disease patients.

On the other hand, the utility indices showed no significant differences between the NA group and the NG group. This finding suggested that, no matter which drug was administered, brand name drug or its GE drug, it produced no significant differences in the QOL of Alzheimer's disease patients. In addition, since no significant differences in utility indices were assessed between the AA and AG groups, we concluded that the patients' QOL was maintained, even if the brand name drug was changed to its GE drug.

\section{Estimated Effects of Donepezil Hydrochloride on Quality-Adjusted Life Years (QALY)}

In this study, the groups starting a new drug regimen showed improvements in the average change in QOL that exceeded approximately 0.1 . This figure was the amount of change assessed during a 4-month period, this study's investigation period. Assuming that the value of QOL rose 0.1 as a result of administering a drug for a period of 0.333 years (4 months), the amount of QALY acquired can be calculated as $0.333 \times 0.1 \times 0.5=0.0167$ QALY. In other words, from the changes assessed after four months, the annual change in QALY can be estimated to be 0.0167 QALY. This is to find the area of triangle, which base length is 0.333 year (duration period) and height is 0.1 (difference of QOL score). We cannot assume how the QOL score were changed between two observation timings. Then, we adopted linear interpolation for calculation, which was recommended in Drummond et al. ${ }^{8)}$ The level of willingness-to-pay (WTP) for 1-QALY (one year of survival in a fully healthy state) in Japan has been confirmed to be approximately JPY 6 million (USD 52,174, JPY 115=USD 1). ${ }^{9,10)}$ Therefore, it is possible to estimate the assumed effects of QALY resulting from new regimens of the drug and continuing for 4 months to be approximately JPY 100,000 (USD 870). The drug price of Aricept (orally disintegrating tablet) $5 \mathrm{mg}$, as of March 23, 2014, was JPY 356 (USD 3.1), so it would cost JPY 42,720 (USD 371) for four months; the drug price of the GE drug (D) is JPY 249.2 (USD 2.2), costing JPY 29,904 (USD 260) for four months. These drug prices are much lower than JPY 100,000 (USD 870) (WTP/4 months). In this study, administration of donepezil hydrochloride appeared to be highly promising, cost effective method to increase QOL. Since this value was calculated from drug costs alone, it is a low estimate. There is a possibility for the cost efficiency to be improved further, if influences on medical expenses, other than from drugs, are revealed by further studies.

Community pharmacists will be expected to establish pharmacies to support dementia patients, providing consultations and assistance to improve the QOL.

Our findings suggested that starting a new regimen of donepezil hydrochloride (Aricept or its GE drug) led to improved QOL in Alzheimer's disease patients and that their QOL was maintained, even if a brand name drug was changed to its GE drug. Taken together, these results demonstrated that the GE drug has similar effects to the brand name drug, regardless of beginning, continuing, or switching drug treatments.

\section{REFERENCES}

1. Asada T. Morbidity rate of dementia in urban areas and measures to cope with difficulties of functioning in the daily lives of dementia patients. Japanese Ministry of Health, Labour and Welfare;2013.

2. Ministry of Health, Labor and Welfare (Japan). The document of 54th meeting of the Long-Term Care Insurance Committee, Social Security Council. 2013 [cited 2015 Mar 25]; Available from: www.mhlw.go.jp/stf/shingi/0000033005.html.

3. Naglie G, Tomlinson G, Tansey C, Irvine J, Ritvo P, Black $\mathrm{SE}$, et al. Utility-based quality of life measures in Alzheimer's disease. Qual Life Res 2006;15:631-643.

4. Kawano Y, Asada T, Kinoshita T, Yasuda A, Hachimori A, Inaba $\mathrm{Y}$, et al. A study of the reliability and validity of the Japanese version of EuroQol EQ-5D and VAS in patients with Alzheimer's disease. Jpn J Geriatr Psychiatr 2009;20: 1149-1159.

5. Hachimori A, Kawano Y, Homma A, Asada T, Ikeda M, Yasuda A, et al. Changes in the comprehensivehealth- 
related $Q O L$ of patients with dementia of Alzheimer type (DAT) treated with donepezil and their primary caregivers. Jpn J Geriatr Psychiatr 2009;20:997-1007.

6. Walters SJ, Brazier JE. Comparison of the minimally important difference for two health state utility measures: EQ-5D and SF-6D. Qual Life Res 2005;14:1523-1532.

7. Yasuda A, Kawano Y, Kinoshita T, Utsumi K, Okumura A, Shigenobu $\mathrm{K}$, et al. Changes in the comprehensivehealthrelated QOL of persons with dementia of the Alzheimer type (DAT) treated with donepezil and their primary carers.
Jpn J Geriatr Psychiatr 2011;22:1433-1445.

8. Drummond MF, Schulper MJ, Torrance GW, O'Brien BJ, Stoddart GL. Methods for the economic evaluation of health care programmes. 3rd ed. Oxford:Oxford University Press;2005.

9. Ookusa Y. A research survey on the upper limit of social burden per QALY. Med Soc 2003;13:121-130.

10. Ookusa Y. A study on the maximum amount of WTP vis-à-vis the acquisition of 1-QALY. Med Soc 2006:13:157-165. 